\title{
PHOTON ENGINEERING FOR QUANTUM INFORMATION PROCESSING
}

\author{
A.B. U'REN ${ }^{a}$, K. BANASZEK AND I.A. WALMSLEY \\ Clarendon Laboratory, Oxford University, Oxford, OX1 3PU, UK \\ May 2003
}

\begin{abstract}
We study distinguishing information in the context of quantum interference involving more than one parametric downconversion (PDC) source and in the context of polarization-entangled photon pairs based on PDC. We arrive at specific design criteria for two-photon sources so that when used as part of complex optical systems, such as photon-based quantum information processing schemes, distinguishing information between the photons is eliminated guaranteeing high visibility interference. We propose practical techniques which lead to suitably engineered two-photon states that can be realistically implemented with available technology. Finally, we study an implementation of the nonlinear-sign shift (NS) logic gate with PDC sources and show the effect of distinguishing information on the performance of the gate.
\end{abstract}

Keywords: parametric down-conversion; polarization-entanglement; quantum communication; Bell state measurement; optical quantum computing

\section{Introduction}

Among physical systems that can be used for practical realizations of novel information processing schemes based on intrinsically quantum phenomena, photons are the primary candidates for constituting carriers of quantum information. Applications of photons as "flying qubits" include quantum cryptography, communication between distributed computational nodes in quantum networks, as well as elementary building blocks for registers in all-optical quantum computation.

Using single photons as carriers of qubits in quantum information processing requires, in most cases, that they be made to interact in a controlled coherent way. This is usually facilitated by modal interference, which is implemented in practice with the help of passive linear optical elements such as beam splitters, polarizers, and wave plates. Though the range of interactions that can be realized by these means is restricted, the linear optical approach is at present the most viable one, as nonlinear effects are usually much too weak at the single photon level in travelling-wave configurations. Modal interference is, however, critically sensitive to the spatio-temporal structure of the interfering photons. Great care must therefore be exercised in making sure that no distinguishing information is contained in the interfering photons that would allow one to trace their origin. A common approach to cope with this problem is the implementation of strong spatial and spectral filtering. Such a method, however, reduces the available photon sample, and also contributes deleteriously to the overall detection efficiency. Overcoming the limitations of this approach is currently one of the main challenges in the further development of quantum information processing applications in the photonic domain.

The purpose of this paper is to explore methods for engineering the modal structure of photon sources to ensure optimal performance of quantum information applications. The

${ }^{a}$ A.B. U'Ren's e-mail address is: alfred.uren@physics.ox.ac.uk 
physical process that we will consider is spontaneous parametric down-conversion which has been the primary source of nonclassical optical radiation in recent experiments ranging from implementations of quantum information technologies to fundamental tests of quantum mechanics. Our goal is to develop photon sources that produce photons in well specified single spatio-temporal modes thus eliminating the usual need for filtering: the resulting engineered photons would have identical mode structures, with in principle no distinguishing information residing in any degree of freedom, thus ensuring high visibility interference. We will base our discussion on the analysis of well known interferometers, such as the event-ready version of the Hong-Ou-Mandel interferometer and the Braunstein-Mann Bell state analyzer. We will also analyze the role of distinguishing information in the nonlinear sign-shift gate that constitutes the basic element of the recently proposed scheme for quantum computing based on linear optics [1] 2].

\section{Criteria for the design of photon states}

\subsection{Characterizing entanglement in photon pairs.}

A two-photon PDC state can in general be expressed as a weighted sum of creation operators acting on vacuum as follows:

$$
\begin{array}{r}
|\Psi\rangle=\sum_{\mu_{s}} \sum_{\mu_{i}} \int_{0}^{\infty} d \omega_{s} \int_{0}^{\infty} d \omega_{i} \int d \mathbf{k}_{s}^{\perp} \int d \mathbf{k}_{i}^{\perp} S\left(\omega_{s}, \mathbf{k}_{s}^{\perp}, \mu_{s} ; \omega_{i}, \mathbf{k}_{i}^{\perp}, \mu_{i}\right) \\
\times \hat{a}_{\mathbf{k}_{s}^{\perp} \mu_{s}}^{\dagger}\left(\omega_{s}\right) \hat{a}_{\mathbf{k}_{i}^{\perp} \mu_{i}}^{\dagger}\left(\omega_{i}\right)|\mathrm{vac}\rangle
\end{array}
$$

where the function $S\left(\omega_{s}, \mathbf{k}_{s}^{\perp}, \mu_{s} ; \omega_{i}, \mathbf{k}_{i}^{\perp}, \mu_{i}\right)$ represents the joint two-photon amplitude in terms of the photon degrees of freedom which include: frequency, transverse momentum (with respect to a fixed direction of propagation defined by the pump field) and polarization. The two-photon probability amplitude depends on the form of the electromagnetic field used as a pump in the down-conversion process, and on the so-called phase matching function, defined by the optical properties of the crystal. A useful tool for analyzing the entanglement present in the continuous degree of freedom is to carry out a Schmidt decomposition [3] of the spatio-temporal-polarization state amplitude $S\left(\mathbf{k}_{s}^{\perp}, \omega_{s}, \mu_{s} ; \mathbf{k}_{i}^{\perp}, \omega_{i}, \mu_{i}\right)$ into a sum of products of functions:

$$
S\left(\omega_{s}, \mathbf{k}_{s}^{\perp}, \mu_{s} ; \omega_{i}, \mathbf{k}_{i}^{\perp}, \mu_{i}\right)=\sum_{n} \sqrt{\lambda_{n}} \psi_{n}\left(\omega_{s}, \mathbf{k}_{s}^{\perp}, \mu_{s}\right) \phi_{n}\left(\omega_{i}, \mathbf{k}_{i}^{\perp}, \mu_{i}\right)
$$

where $\omega_{s, i}, \mathbf{k}_{s, i}^{\perp}$ and $\mu_{s, i}$ represent the wavelength, transverse wavevector and polarization of the signal (s) and idler (i) photons. The Schmidt functions $\psi_{n}\left(\omega_{s}, \mathbf{k}_{s}^{\perp}, \mu_{s}\right)$ and $\phi_{n}\left(\omega_{i}, \mathbf{k}_{i}^{\perp}, \mu_{i}\right)$ can be thought of as the basic building blocks of entanglement in the sense that if the signal photon is determined to be described by a function $\psi_{n}$, we know with certainty that its idler sibling is described by the corresponding function $\phi_{n}$. The probability of finding this specific pair of modes is given by the parameter $\lambda_{n}$, assumed to be real and nonnegative. In general, obtaining such a Schmidt decomposition entails solving certain integral equations [3]. By approximating the sinc function in the phase matching function as a Gaussian it becomes possible to carry out the decomposition analytically in certain cases [4] (see appendix A). It is found that the resulting entanglement does not necessarily involve the infinitely many degrees 
of freedom in the frequency-space-polarization continuum with typically only a finite number of Schmidt modes having appreciable eigenvalue magnitudes. The amount of entanglement can be conveniently quantified by the cooperativity parameter [5] defined in terms of the Schmidt eigenvalues as:

$$
K=\frac{1}{\sum_{k} \lambda_{k}^{2}}
$$

The value of $K$ gives an indication of the number of active Schmidt mode pairs, which in turn is a measure of how much entanglement (including spectral, spatial and polarization degrees of freedom) is present in the photon pairs. A two-photon state for which the cooperativity assumes its minimum allowed value $K=1$ represents a state in which there is a single pair of Schmidt modes and therefore exhibits no spectral entanglement.
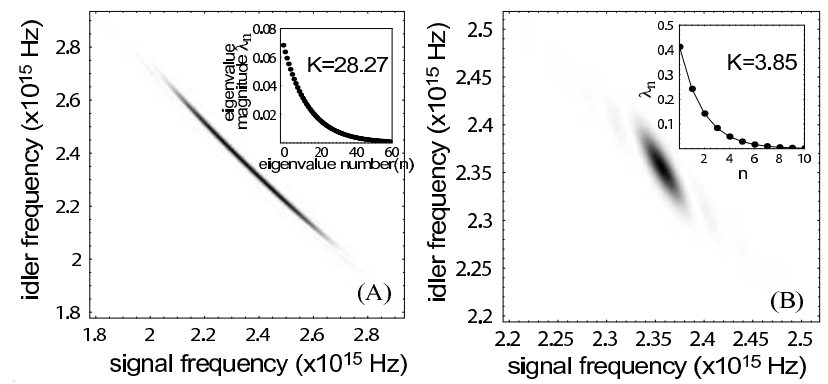

Fig. 1. Joint spectral intensity of ultrafast-pumped (15 nm bandwidth) PDC centered at $800 \mathrm{~nm}$ from a $1 \mathrm{~mm}$ long BBO crystal. (A) shows an example of a two photon state involving noncollinear type I phase matching and (B) shows a two photon state in the case of collinear type II phase matching. Note that type I PDC has a higher degree of spectral entanglement as quantified by the value of the cooperativity parameter $K$. Such sources with high $K$ are not suitable for a number of multiple-source experiments.

Let us turn our attention to actual examples of two-photon states produced by PDC. We will consider non-collinear type-I (eoo) and collinear type-II (eoe) PDC processes in a betabarium-borate (BBO) crystal pumped by a train of ultrafast pulses. Note that for a Schmidt decomposition of a PDC state to have physical meaning the signal and idler photons must be distinguishable; in the case of non-collinear type-I PDC this is ensured by distinct spatial modes while in the case of type-II PDC this is ensured by the orthogonal polarizations. For both of these cases, once we select specific directions of propagation for the down-converted photons, the joint two-photon probability amplitude becomes a function of only the two frequencies $\omega_{s}$ and $\omega_{i}$ and it can be written as follows [8]: $S\left(\omega_{s}, \omega_{i}\right)=\alpha\left(\omega_{s}+\omega_{i}\right) \phi\left(\omega_{s}, \omega_{i}\right)$ where $\alpha\left(\omega_{s}+\omega_{i}\right)$ is the Fourier transform of the temporal pump amplitude and $\phi\left(\omega_{s}, \omega_{i}\right)$ is the crystal phase matching function. The pump envelope function may be modelled as a Gaussian $\alpha\left(\omega_{s}+\omega_{i}\right)=\exp \left[-\left(\omega_{s}+\omega_{i}-2 \omega_{0}\right)^{2} / \sigma_{p}^{2}\right]$ with a bandwidth $\sigma_{p}$ and which is centered at $2 \omega_{0}$. The phase matching function is given as $\phi\left(\omega_{s}, \omega_{i}\right)=\operatorname{sinc}(L \Delta k / 2)$ where $L$ is the crystal length and $\Delta k=k_{p}\left(\omega_{p}\right)-k_{s}\left(\omega_{s}\right)-k_{i}\left(\omega_{i}\right)$ incorporates the dispersion characteristics of the crystal. In Fig. 1 we see that for typical experimental parameters both type I and type II PDC, though particularly the former, exhibit large spectral entanglement, as quantified by 
the large value of $K$.

Is a high value of $K$ such as is obtained from a typical PDC crystal desirable? The answer to this question depends on what experiment we wish to perform. In the following two sections we will explore the specific requirements on two-photon states necessary to guarantee high visibility interference in two cases: i) multiple-crystal interferometry experiments and ii) experiments relying on polarization entangled photon pairs.

\subsection{Criteria for the design of photon states to be used in multiple-crystal inter- ferometry.}

A basic requirement necessary for the successful implementation of many photon-based quantum computation protocols is the availability of multiple-photon entangled states. In this section we explore the experimental challenges likely to be faced when synthesizing such a multiple-photon state from a number of two-photon sources pumped synchronously with an ultrashort pulsed pump. Employing such an approach, distinguishing information implicit in timing can be limited to the pulsed pump temporal duration. Unfortunately, distinguishing information may remain in the other degrees of freedom. Filtering is a convenient way often used in experiments to accurately define spatio-temporal modes from PDC crystals and therefore eliminate distinguishing information. Filtering, however, unfortunately leads to a prohibitive reduction of the usable photon production rate. Eliminating spectral distinguishing information without resorting to filtering, in the context of multiple-crystal interferometry, is the theme of this section. That filtering is undesirable is manifest in that a multiple photon source based on several PDC sources necessitates the simultaneous emission of photon pairs from all sources used, which unfortunately occurs with an exponentially decreasing probability as the number of sources is increased (if the probability of obtaining a random event from one source is $p$ the probability of $N$ simultaneous events from $N$ sources is $p^{N}$ ). Though it is rather difficult to view such an approach based on PDC as a practical route towards fully scalable all-optical quantum computation, it can be reasonably expected that this process can be applied in small-scale quantum circuits used for example in quantum communications.

Solid state sources of single photons have recently been reported to exhibit enough indistinguishability for quantum interference effects to be observed [6]. Such sources, however, require a relatively complex apparatus including the need for cryostatic cooling. On the other hand, it is difficult to direct emission to well-defined spatial modes, making collection efficiencies of the produced photons quite limited. PDC-based sources of indistinguishable photons, to be discussed in this section, operate at room temperature and require little besides a $\chi^{(2)}$ crystal, focused pump beam and apertures to define the output spatial modes.

Under what conditions are photons emitted by different sources indistinguishable? The well-known Mandel dip experiment [10] is a good platform on which to test the concept of indistinguishability. For the classical Hong-Ou-Mandel interferometer (HOMI) it turns out that the fourth-order interference visibility depends only on the degree to which the joint spectral amplitude is symmetric [symmetry defined for our purposes as $S\left(\omega_{s}, \omega_{i}\right)=S\left(\omega_{i}, \omega_{s}\right)$ where $S\left(\omega_{s}, \omega_{i}\right)$ is the joint spectral amplitude], assuming that the minimum of the interference dip occurs for zero time delay between the interfering photons. The physical reason for this is that the two Feynman paths contributing to a coincidence event $\left(R_{x} R_{x}\right.$ path: $\omega_{a}$ in the idler arm reflected and $\omega_{b}$ in the signal arm reflected. $T_{x} T_{x}$ path: $\omega_{a}$ in the signal arm transmitted 
and $\omega_{b}$ in the idler arm transmitted, where $\omega_{a}$ and $\omega_{b}$ are the frequencies of the photons registered by the respective detectors) become distinguishable if the symmetry condition is not fulfilled. Thus, for the Mandel dip experiment a large value of $K$ does not constitute a limitation, as indeed has been shown experimentally by the nearly $100 \%$ interference visibility obtained for type-I PDC in several experiments both in the $\mathrm{CW}$ and pulsed pumped regimes.

Let us now discuss a two-PDC crystal interference experiment in an event-ready HOMIlike arrangement. Consider two PDC crystals as shown in Fig. 2 and a HOMI arrangement interfering the idler from the first crystal with the signal from the second crystal, the remaining two channels being used as triggers. The experiment would consist of monitoring quadruple coincidences on $D 1$ through $D 4$ while scanning the delay $\tau$. If both PDC crystals are identical and described by a joint spectral amplitude function $f\left(\omega_{s}, \omega_{i}\right)$, the four-fold coincidence rate $R_{c}(\tau)$ may be shown to be proportional to 9 :

$$
\begin{gathered}
R_{c}(\tau) \propto \int_{0}^{\infty} \int_{0}^{\infty} \int_{0}^{\infty} \int_{0}^{\infty} d \omega_{1} d \omega_{2} d \omega_{3} d \omega_{4} f\left(\omega_{1}, \omega_{2}\right) f\left(\omega_{3}, \omega_{4}\right)\left[f^{*}\left(\omega_{1}, \omega_{2}\right) f^{*}\left(\omega_{3}, \omega_{4}\right)\right. \\
\left.-e^{i\left(\omega_{1}-\omega_{3}\right) \tau} f^{*}\left(\omega_{1}, \omega_{4}\right) f^{*}\left(\omega_{3}, \omega_{2}\right)\right]
\end{gathered}
$$

We can see from Eq. (4) that the visibility becomes unity if at $\tau=0$, i.e. at zero delay between the two interferometer paths, the coincidence rate vanishes. A sufficient condition for this to occur is that the integrand itself vanish at $\tau=0$, which leads to the condition

$$
f\left(\omega_{1}, \omega_{2}\right) f\left(\omega_{3}, \omega_{4}\right)=f\left(\omega_{1}, \omega_{4}\right) f\left(\omega_{3}, \omega_{2}\right)
$$

for any $\omega_{1}, \omega_{2}, \omega_{3}$ and $\omega_{4}$.

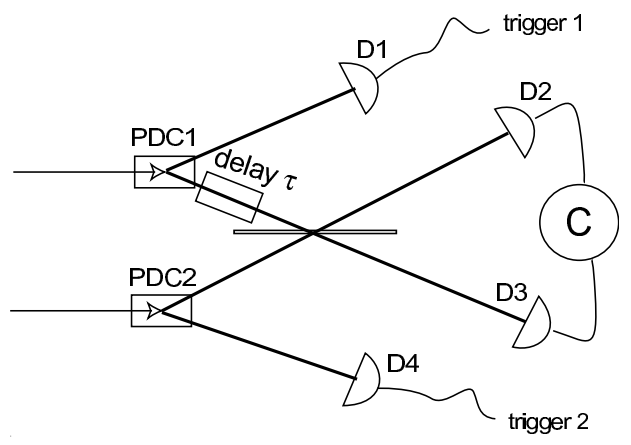

Fig. 2. Two PDC crystal HOMI arrangement. This is an example of an experiment where interference visibility is reduced if the photon pairs are spectrally correlated. Spectral correlation leads to distinguishing information between the two photons which inhibits interference.

Unlike in the one-crystal Mandel dip experiment, symmetry in the function $f\left(\omega_{s}, \omega_{i}\right)$ does not help in fulfilling this condition. However, it may be seen that such a condition is met if the function $f\left(\omega_{s}, \omega_{i}\right)$ is factorizable i.e. if functions $p(\omega)$ and $q(\omega)$ exist such that $f\left(\omega_{s}, \omega_{i}\right)=p\left(\omega_{s}\right) q\left(\omega_{i}\right)$. For such a factorizable state there is a single Schmidt mode pair i.e. $K=1$, which means that there is no spectral entanglement. The nature of distinguishing 
information is therefore different in the case of multiple PDC source experiments to that of single source experiments. Whereas in the former distinguishing information resides in the spectral entanglement, in the latter it resides in the joint spectral amplitude asymmetry.

By what physical mechanisms can we obtain such a factorizable state? Perhaps the simplest way is by making use of spectral and/or spatial filtering. A recent experiment by Mlynek et al. [7 demonstrates the way in which distinguishing information can be eliminated via filtering but at the cost of extremely low resulting production rates. To understand how this works, consider a simplified type-I source where the joint spectral amplitude is approximated by a Gaussian (i.e. we neglect the secondary peaks of the sinc function and also neglect the effects of dispersion) and is multiplied by a Gaussian spectral filter function:

$$
f\left(\nu_{s}, \nu_{i}\right)=A \exp \left[-2\left(\nu_{s}^{2}+\nu_{i}^{2}\right)\left(\frac{1}{\sigma_{F}^{2}}+\frac{1}{\sigma^{2}}\right)-\frac{2 \nu_{s} \nu_{i}}{\sigma^{2}}\right]
$$

where $A$ is a normalization constant, $\nu_{j}=\omega_{j}-\omega_{0}$ [with $\left.j=s, i\right], \sigma_{F}$ is the width of the Gaussian spectral filter and $\sigma$ is a resulting width of the product of the pump envelope and phase matching functions. Substituting Eq. (6) into Eq. (4) and carrying out the integrals we obtain the coincidence rate function:

$$
R_{c}(\tau) \propto R_{0}\left[1-V \exp \left(-\frac{\sigma^{2} \sigma_{F}^{2} \tau^{2}}{8\left(\sigma_{F}^{2}+\sigma^{2}\right)}\right)\right]
$$

which represents an interference dip with a temporal width proportional to $\sqrt{\sigma^{2}+\sigma_{F}^{2}} /\left(\sigma \sigma_{F}\right)$ and a visibility $V$ given by:

$$
V=\sqrt{1-\frac{\sigma_{F}^{4}}{\left(\sigma_{F}^{2}+\sigma^{2}\right)^{2}}}
$$

The normalized count rate, $R_{0}$ (i.e. the coincidence rate in the limit $\tau \rightarrow \infty$ ) is given by:

$$
R_{0}=\frac{2 \sigma_{F}^{2}}{2 \sigma_{F}^{2}+\sigma^{2}}
$$

which has a value between 0 and 1 . For a fixed value of $\sigma$, the filtering strength determines the value of $R_{0}$, which may be seen to vanish for strong filtering $\left(\sigma_{F} \ll \sigma\right)$ and gives unity in the limit of no filtering $\left(\sigma_{F} \gg \sigma\right)$.

In the strong filtering regime (i.e. $\sigma_{F} \ll \sigma$ ), Eq. (8) predicts that the visibility reaches unity. In this case the joint spectral amplitude reduces to $f\left(\nu_{s}, \nu_{i}\right)=\exp \left[-2\left(\nu_{s}^{2}+\nu_{i}^{2}\right) / \sigma_{F}^{2}\right]$, which is factorizable [and thus fulfils Eq. (5)] with identical $p(\nu)$ and $q(\nu)$ functions given by $p(\nu)=q(\nu)=\exp \left(-2 \nu^{2} / \sigma_{F}^{2}\right)$. Spectral filtering can be straightforwardly carried out experimentally with suitable interference filters readily available. Filtering, however, has the unfortunate consequence that the count rate is prohibitively reduced by rejecting most of the photon pairs (and retaining only those without distinguishing information). This is illustrated in Fig. [3(A) which shows the relationship between the visibility [see Eq. [8] ] in the two-crystal HOMI and the expected count rate [see Eq. (9] ] with each point along the curve corresponding to a different filtering strength. Fig. B(B) depicts the relationship between the expected two-crystal visibility and the cooperativity parameter [assuming that both PDC 
sources are characterized by the simplified type-I spectral amplitude given in Eq. [6]). Note that unit visibility is obtained only in the limiting case of a single active Schmidt mode $K=1$, that is in the case where each PDC source exhibits no spectral correlation.
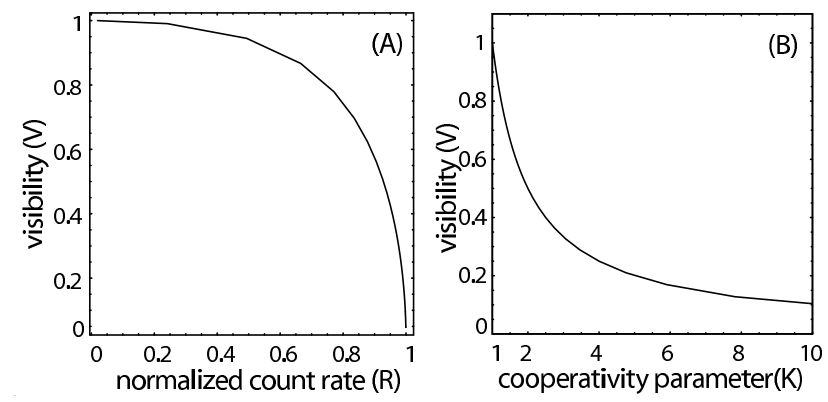

Fig. 3. (A) depicts the tradeoff between the interference visibility and the count rate expected in a two-crystal HOMI setup for different filtering strengths. Unit visibility is obtained only in the limit where the filtering bandwidth approaches zero. (B) depicts the relationship between the number of active Schmidt modes, the cooperativity parameter, and the visibility. Interference is reduced if there is more than one mode. In generating this plot Eqs. A.3 and A.4 from the appendix were used. For the plots in this figure, a type-I simplified source with $\sigma=4 \times 10^{13} \mathrm{~Hz}$ was assumed while the value of $\sigma_{F}$ was allowed to take a range of values.

\subsection{Criteria for the design of polarization entangled photon pairs}

The polarization of light fields can be readily controlled using, for example, wave plates and polarizers. This is in marked contrast with the case of other photonic degrees of freedom, such as frequency, for which it is rather more difficult to achieve a comparable level of control. It is therefore not surprising that most recent experiments exploring issues of entanglement have relied on polarization entanglement. A number of reliable methods for generating polarization entangled photon pairs have been proposed and implemented [12, 13].

As in any interference experiment, a pre-condition for high interference visibility is that distinguishing information between the interfering pathways is eliminated, even in those degrees of freedom which are not of primary interest for the experiment in question. This applies to the generation of polarization-entangled photon pairs, where even though polarization is the degree of freedom of interest, it is crucial to appropriately engineer the spectral properties of the photon pairs to eliminate any spectral distinguishing information[11.

We will illustrate the importance of this point by studying the Braunstein-Mann Bell state analyzer [14. The apparatus is displayed in figure 4(A) where BS is a 50:50 beam-splitter and PBS1 and PBS2 are polarizing beam-splitters. This experiment serves as a building block for a large class of experiments relying on Bell state measurement, such as entanglement swapping and teleportation. Employing such an analyzer, the experimenter can make inferences about the state of the incoming photon pair from the firing pattern of the four detectors. Likewise, knowing the state of the incoming photon pair, inferences can be made about what the firing pattern will be. For instance, assuming that there is zero temporal delay between the two photons $(\tau=0)$, if $\alpha$ and $\gamma$ or $\beta$ and $\delta$ fire we then know with certainty that the incoming state is the singlet state $\left|\psi^{(-)}\right\rangle_{a b}=2^{-\frac{1}{2}}\left(|H V\rangle_{a b}-|V H\rangle_{a b}\right)$ [where $H$ and $V$ refer to horizontal and 
vertical polarization and $a$ and $b$ refer to the two spatial modes]. Likewise, if the incoming state is $\left|\psi^{(+)}\right\rangle_{a b}=2^{-\frac{1}{2}}\left(|H V\rangle_{a b}+|V H\rangle_{a b}\right)$, we can infer with certainty that either $\alpha$ and $\beta$ or $\delta$ and $\gamma$ will fire. Considering the fact that the Bell state analyzer relies on quantum interference, we would expect that if the two-photon state used exhibits spectral distinguishing information, the correlation between the incoming two-photon state and the firing pattern will no longer be perfect. We will compare the behavior of the Bell-state analyzer with that of the apparatus shown in Fig 4 (B), which measures the polarization correlations present in a polarization-entangled two-photon state.
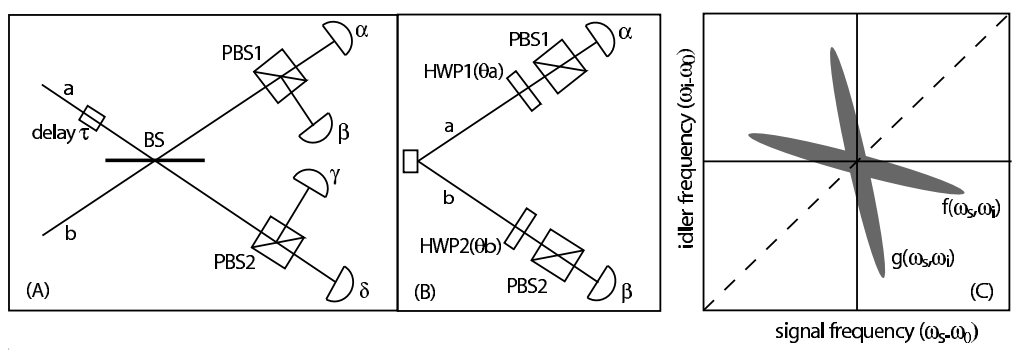

Fig. 4. (A) Braunstein-Mann Bell state analyzer. (B) Polarization correlation experiment. (C) Graphical representation of the condition for optimal performance.

We begin our analysis by considering a maximally-entangled polarization Bell state with the spectral component of the state included in explicit form:

$$
\left|\psi^{ \pm}\right\rangle=\frac{1}{\sqrt{2}} \int_{0}^{\infty} \int_{0}^{\infty} d \omega_{1} d \omega_{2}\left[f\left(\omega_{1}, \omega_{2}\right) a_{H}^{\dagger}\left(\omega_{1}\right) b_{V}^{\dagger}\left(\omega_{2}\right) \pm g\left(\omega_{1}, \omega_{2}\right) a_{V}^{\dagger}\left(\omega_{1}\right) b_{H}^{\dagger}\left(\omega_{2}\right)\right]|\mathrm{vac}\rangle
$$

where $a$ and $b$ refer to two distinct spatial modes, and where the functions $f\left(\omega_{1}, \omega_{2}\right)$ and $g\left(\omega_{1}, \omega_{2}\right)$ are each normalized such that the integral over both arguments of the modulus squared of the function is unity. In order to study the performance of the Bell state analyzer, we detect the whole output from each port of the beam-splitter in figure 4 (A) i.e. we remove the two polarizing beamsplitters and place a detector in each output mode. If the Bell state analyzer operates ideally, as described above, the singlet $\left|\psi^{(-)}\right\rangle$state yields a unit coincidence rate while the $\left|\psi^{(+)}\right\rangle$yields a zero coincidence rate. On the other hand, calculating the coincidence rate assuming a general incoming state given as in eq. (10), we obtain the result:

$$
R_{c}^{ \pm}(\tau)=\frac{1}{4} \int_{0}^{\infty} \int_{0}^{\infty} d \omega_{1} d \omega_{2}\left|f\left(\omega_{1}, \omega_{2}\right) \mp \mathrm{e}^{i\left(\omega_{1}-\omega_{2}\right) \tau} g\left(\omega_{2}, \omega_{1}\right)\right|^{2}
$$

where the $+/$ - on the left hand side refers to a $\psi^{(+)} / \psi^{(-)}$incoming state. Because the integrand in eq. (11) is non-negative, the necessary and sufficient condition to obtain $R_{c}^{+}=0$ and $R_{c}^{-}=1$ at $\tau=0$ (i.e. the desired behavior) is that:

$$
g\left(\omega_{1}, \omega_{2}\right)=f\left(\omega_{2}, \omega_{1}\right)
$$


Fig. $4(\mathrm{C})$ depicts the condition in eq. (12) graphically: the two spectral amplitude functions must be the specular image of each other across the line $\omega_{1}=\omega_{2}$. In order to gain a physical understanding of this condition we consider the Feynman alternatives leading to obtaining a coincidence event. Suppose that the photon pair is described by the first component of the Bell state, i.e. the amplitude containing $f\left(\omega_{1}, \omega_{2}\right)$ and that the two photons are reflected at the beam splitter. Such an event [left diagram in Fig. 囵(A)] is indistinguishable (meaning having an identical detection pattern) from the event where the photon pair is described by the second amplitude with reversed frequency arguments i.e. $g\left(\omega_{2}, \omega_{1}\right)$, and where the two photons are transmitted at the beam splitter [see right diagram in Fig [5(A)]. For this system, there is a second set of two pathways as shown in Fig. 5(B) which may lead to a coincidence. For unit interference visibility to occur, the two pathways in each set must be indistinguishable from each other. Fig. [5] shows schematically that for both sets of pathways, indistinguishability is guaranteed (for $\tau=0$ ) if the condition in Eq. (12) is fulfilled.

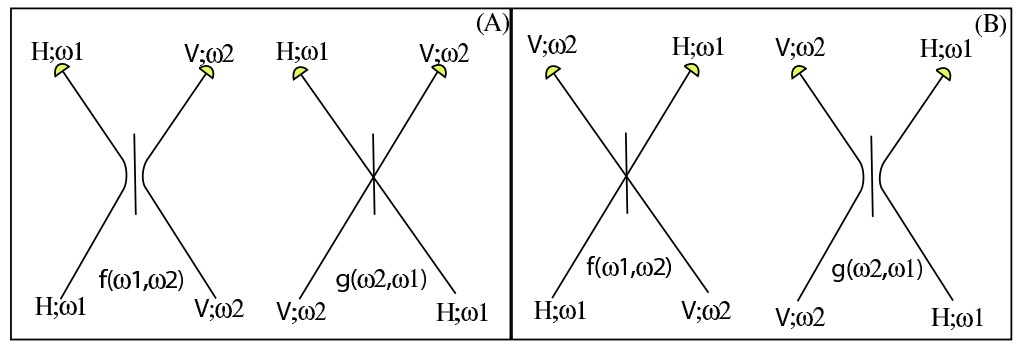

Fig. 5. The two Feynman alternatives shown in each box are indistinguishable from each other.

Consider now the apparatus in Fig. 廿(B), which is the standard method used has been used to test whether a photon pair is polarization-entangled. Assuming that the incoming state is given as in eq. (10), the coincidence rate as a function of the polarization rotation angles in each arm, $\theta_{a}$ and $\theta_{b}$, may be shown to be given by:

$$
R_{c}^{ \pm}\left(\theta_{a}, \theta_{b}\right)=\int_{0}^{\infty} \int_{0}^{\infty} d \omega_{1} d \omega_{2}\left|\cos \theta_{a} \sin \theta_{b} f\left(\omega_{1}, \omega_{2}\right) \pm \sin \theta_{a} \cos \theta_{b} g\left(\omega_{1}, \omega_{2}\right)\right|^{2}
$$

The resulting necessary and sufficient condition to obtain unit visibility fringes [in which case the coincidence rate reduces to $\left.R_{c}^{ \pm}\left(\omega_{a}, \omega_{b}\right)=\sin ^{2}\left(\theta_{a} \pm \theta_{b}\right)\right]$ is:

$$
f\left(\omega_{1}, \omega_{2}\right)=g\left(\omega_{1}, \omega_{2}\right)
$$

Note that this condition, which says that the two amplitudes should be identical, is different from that derived for the Bell state analyzer experiment [see eq. (12)]. The fact that these conditions differ means that depending on the kind of experiment to be performed the polarization-entangled state to be used should entail differently engineered spectral properties.

Is it possible to fulfil both conditions simultaneously? Together, the two conditions reduce to the requirement that the quantum amplitudes be identical and symmetric i.e. $f\left(\omega_{1}, \omega_{2}\right)=$ $f\left(\omega_{2}, \omega_{1}\right)=g\left(\omega_{1}, \omega_{2}\right)$. Generating a polarization-entangled state using two type-I cascaded 
crystals [13, the two amplitudes $f\left(\omega_{1}, \omega_{2}\right)$ and $g\left(\omega_{1}, \omega_{2}\right)$ are each symmetric and can be made identical, even in the case where a pulsed pump is used, if the pump is pre-compensated as described by Nambu et al. [15]. Therefore, this method for generating polarization-entangled states has the important property that it can be made to fulfil both conditions [Eq. (12) and Eq. (14)], so that the same source may be used successfully (without the need for spectral filtering) for the polarization-correlation experiment [Fig. 4(A)] and for the Bell state analyzer experiment [Fig. 4(B)].

Polarization-entangled photon pairs may also be synthesized using the modes defined by the intersections of the "e"-ray and "o"-ray cones in type II PDC 12. It may be shown that this scheme produces a polarization-entangled state such that the condition in eq. (12) is fulfilled. This means that such a state is well-suited for Bell state-measurement experiments [e.g. the Bell state analyzer in Fig. [4(A)] while it is not well suited for the polarization correlation experiment [Fig. 4(B)]. In a recent experiment making use of this type II source [17. 16], one of the two spatial modes is subjected to a $90^{\circ}$ polarization rotation prior to be being combined at a beamsplitter with the other mode. The authors show that the two output modes from the beamsplitter are in a polarization-entangled state which yields near unit visibility, without resorting to spectral filtering, in a polarization correlation experiment such as the one shown in Fig. 目(B). We can understand the results of Kim et al. in terms of the conditions in Eq. (12) and Eq. (14). The half-wave plate and beamsplitter accomplish turning a state obeying $f\left(\omega_{1}, \omega_{2}\right)=g\left(\omega_{2}, \omega_{1}\right)$ into a state obeying $f\left(\omega_{1}, \omega_{2}\right)=g\left(\omega_{1}, \omega_{2}\right)$. The resulting state, however, is no longer well suited for Bell-measurement experiments.

For a polarization-entangled photon pair based on type-II PDC to be simultaneously optimized for both kinds of experiments (Bell state analysis and polarization correlation), the signal and idler photons need to fulfil the so-called group velocity matching condition [18, 4] which for a given $\chi^{(2)}$ material is met at a specific (usually long, $>1 \mu \mathrm{m}$ ) wavelength. For BBO, for example, such a condition is met at a central PDC wavelength of $1.51 \mu \mathrm{m}$ at which singlephoton detection is not technologically well-developed. It would therefore appear that it is possible to optimize an experimentally-feasible polarization-entangled photon pair generated via type-II PDC (which does not fulfil the group velocity matching condition) for one of the two types of experiments discussed above, but not for both simultaneously. There are in fact two ways in which the amplitudes may be made identical and symmetric, and therefore be made to fulfil both conditions. The first is by pumping the type-II source with a CW pump: in the limit of zero pump bandwidth, the pump envelope function which is always symmetric dominates over the phase matching function to determine the overall spectral amplitude. It is also possible to symmetrize the amplitudes by placing a (symmetric) interference filter with a narrow enough bandwidth and an appropriately chosen central bandpass frequency at each of the two spatial modes. The latter, of course, at the cost of a sharp reduction in the production rate of photon pairs. We therefore conclude that an ultrafast-pumped spectrally unfiltered and non-group-velocity-matched type-II polarization-entangled source cannot be simultaneously optimized for both kinds of experiments, in contrast with a two type-I cascaded crystal source.

As discussed above, a sufficient condition for a polarization-entangled state to be simultaneously optimized for Bell state measurement and for polarization correlation experiments (see Fig. (4) is that the joint spectral amplitude be symmetric i.e. $f\left(\omega_{1}, \omega_{2}\right)=f\left(\omega_{2}, \omega_{1}\right)$. This requirement is fulfilled in the specific case where the joint spectral amplitude exhibits no spec- 
tral correlations (see also Sec. 3.1). Here we investigate the possible advantages of engineering polarization entangled two-photon states so that they are also spectrally uncorrelated.

Why would such a state be useful? In order to answer this question we analyze the $\left|\phi^{ \pm}\right\rangle$ state such as would be produced by a cascaded type-I polarization-entangled PDC source 13 . 15]:

$$
\left|\phi^{ \pm}\right\rangle=\frac{1}{\sqrt{2}} \int_{0}^{\infty} \int_{0}^{\infty} d \omega_{1} d \omega_{2} f\left(\omega_{1}, \omega_{2}\right)\left[a_{H}^{\dagger}\left(\omega_{1}\right) b_{H}^{\dagger}\left(\omega_{2}\right) \pm a_{V}^{\dagger}\left(\omega_{1}\right) b_{V}^{\dagger}\left(\omega_{2}\right)\right]|\mathrm{vac}\rangle
$$

where the joint spectral amplitude is assumed to be symmetric: $f\left(\omega_{1}, \omega_{2}\right)=f\left(\omega_{2}, \omega_{1}\right)$. Suppose now that the spectral amplitude is uncorrelated (i.e. has a cooperativity parameter value $K=1)$ such that functions $p(\omega)$ and $q(\omega)$ exist yielding $f\left(\omega_{1}, \omega_{2}\right)=p\left(\omega_{1}\right) q\left(\omega_{2}\right)$. The two-photon state [Eq. (15)] becomes:

$$
\left|\phi^{ \pm}\right\rangle=\frac{1}{\sqrt{2}}\left(c_{H}^{\dagger} d_{H}^{\dagger} \pm c_{V}^{\dagger} d_{V}^{\dagger}\right)|\mathrm{vac}\rangle
$$

where:

$$
\begin{aligned}
c_{H, V}^{\dagger} & =\int_{0}^{\infty} d \omega p(\omega) a_{H, V}^{\dagger}(\omega) \\
d_{H, V}^{\dagger} & =\int_{0}^{\infty} d \omega q(\omega) b_{H, V}^{\dagger}(\omega)
\end{aligned}
$$

We see from Eq. (16) that a spectrally uncorrelated polarization-entangled two-photon state can be written in terms of "effective" creation operators, in which the spectral degree of freedom no longer plays a role once integrated out [see Eq. (17) and Eq. (18)]. The fact that the spectral degree of freedom is no longer present means that spectral indistinguishability is guaranteed in any interference experiment, making this an ideal source of polarization entangled photons. Operators $a_{H, V}^{\dagger}$ corresponding to spatial mode $a$ are translated into effective operators $c_{H, V}^{\dagger}$ [see Eq. (17)] while operators $b_{H, V}^{\dagger}$ corresponding to spatial modes $b$ are translated into effective operators $d_{H, V}^{\dagger}$ [see Eq. (18)]. We have thus shown that if polarization-entangled photon pairs are appropriately engineered, the spectral degree freedom can be altogether eliminated.

The use of the spectrally engineered polarization-entangled photon pairs described in this section together with stimulated PDC as described by De Martini and co-workers in Ref. [19] and Lamas-Linares and co-workers in Ref. [20], opens up the possibility of generating photon pairs exhibiting more complex kinds of entanglement while maintaining spectral indistinguishability. The availability of such states may be of crucial importance for realizing quantum computation schemes.

\section{Engineering photon pairs for quantum information.}

\section{$3.1 \quad$ Engineering states with no spectral correlations}

Our approach is to investigate ways to engineer the state at the source in order to obtain a factorizable state thus eliminating the need for filtering. One such approach is described in 
Ref. 4] where it is shown that for degenerate collinear type-II phase matching, and for an appropriate choice of material, central PDC wavelength, crystal length and pump bandwidth, it is indeed possible to obtain such a spectrally uncorrelated state. This scheme makes use of the fact that in some spectral regions and for some materials the phase matching function contours have a positive slope that can counteract the negative slope of the pump envelope function. Unfortunately, for most common $\chi^{(2)}$ materials, this positive slope occurs at longer wavelengths $(>1 \mu \mathrm{m})$, where single-photon detectors are not technologically well developed. For example in the case of $\mathrm{BBO}$ an uncorrelated state is possible for central PDC wavelengths between $1.15 \mu \mathrm{m}$ and $1.92 \mu \mathrm{m}$.

Here we present a novel method for generating spectrally uncorrelated pairs making use of non-collinear, degenerate type-I PDC in bulk crystals and which exploits the transverse momentum of the photons. This approach requires the ability to specify accurately the crystal length and the beam diameter at the beam waist (i.e. the focusing strength) and requires the spatial modes exiting the crystal to be accurately defined for example with pinholes or fibers. An important additional requirement is an ultrafast pulsed pump. Fig. [6 outlines the experimental setup. Unlike the approach in Ref. 4, this method can be made to work at any PDC central wavelength where phase matching is possible, in particular at those where silicon based single-photon counting modules work efficiently.

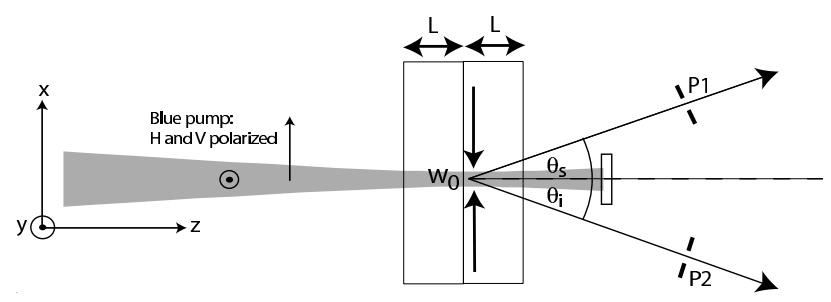

Fig. 6. Uncorrelated photon pair generation through non-collinear type-I PDC in a bulk typeI crystal making use of a Gaussian beam pump. Pinholes $P 1$ and $P 2$ are required in order to accurately define two spatial modes.

We begin by extending the phase matching function to the case where a Gaussian beam, rather than a plane wave, is used as pump field. In this case the electric field amplitude is given by:

$$
\alpha(x, y, z ; k)=\frac{1}{1+\frac{2 i z}{k w_{0}^{2}}} \exp \left(-\frac{x^{2}+y^{2}}{w_{0}^{2}\left(1+\frac{2 i z}{k w_{0}^{2}}\right)}\right) e^{i k z}
$$

where $w_{0}$ is the beam diameter at the beam waist. Following the procedure sketched out in Ref. [8], we obtain for the phase matching function:

$$
\phi\left(\Delta k_{z}, \Delta k^{\perp}\right) \propto \exp \left(-\frac{\left(\Delta k^{\perp}\right)^{2} w_{0}^{2}}{4}\right) \operatorname{sinc}\left[\left(\frac{\left(\Delta k^{\perp}\right)^{2}}{4 k}-\frac{\Delta k_{z}}{2}\right) L\right]
$$

where $\Delta k^{\perp}$ refers to the transverse momentum along the $x-z$ plane (see Fig. 6) so that it is a scalar quantity. Making the approximation $\operatorname{sinc}(x) \approx \exp \left(-\gamma x^{2}\right)\left(\right.$ with $\left.\gamma=.193 \ldots{ }^{\mathrm{a}}\right)$ and the

a The value $\gamma=.193 \ldots$ is obtained by letting the Gaussian function used have the same FWHM width as the sinc function being approximated 
further approximation $w_{0} / L \gg \sqrt{\gamma}\left(\Delta k^{\perp} / k\right)^{2}$ (or equivalently $w_{0} / L \gg \sqrt{\gamma} \sin ^{2} \theta$ where $\theta$ is the propagation angle of the downconverted photons) which sets an upper limit on the focusing strength, the transverse momentum and longitudinal momentum contributions to the phase matching function can be factorized: $\phi\left(\Delta k_{z}, \Delta k_{\perp}\right) \propto \phi_{z}\left(\Delta k_{z}\right) \phi^{\perp}\left(\Delta k^{\perp}\right)$ where:

$$
\begin{aligned}
\phi_{z}\left(\Delta k_{z}\right) & =\exp \left(-\frac{\gamma \Delta k_{z}^{2} L^{2}}{4}\right) \\
\phi^{\perp}\left(\Delta k^{\perp}\right) & =\exp \left(-\frac{\left(\Delta k^{\perp}\right)^{2} w_{0}^{2}}{4}\right)
\end{aligned}
$$

The joint spectral amplitude can now be written as $S\left(\omega_{s}, \omega_{i}\right)=\alpha\left(\omega_{s}+\omega_{i}\right) \phi_{z}\left(\Delta k_{z}\right) \phi^{\perp}\left(\Delta k^{\perp}\right)$, where $\alpha\left(\omega_{s}+\omega_{i}\right)$ is the pump envelope function.

Let us restrict our attention to the case where the photons are emitted along the $x-z$ plane (see Fig. 6), so that the direction of propagation of a given photon can be described with the polar angle (defined within the crystal) $\theta$ (and having zero azimuthal angle $\phi=0$ ). We assume that the pump propagates such that $\theta_{p}=0$ in which case conservation of transverse momentum dictates that $\theta_{s}=-\theta_{i}=\theta$. Performing a Taylor expansion and neglecting second and higher order terms, the longitudinal and transverse phase mismatch are then given by:

$$
\begin{aligned}
\Delta k_{z} & =\Delta k_{z}^{(0)}+\left(k_{p}^{\prime}-k^{\prime} \cos \theta\right)\left(\nu_{s}+\nu_{i}\right) \\
\Delta k^{\perp} & =-k^{\prime} \sin \theta\left(\nu_{s}-\nu_{i}\right)
\end{aligned}
$$

where $\nu_{j}=\omega_{j}-\omega_{0}$ with $j=s, i$. All wavevector amplitudes and their frequency derivatives are evaluated at $\omega_{0}$ (or $2 \omega_{0}$ in the case of $k_{p}$ and $k_{p}^{\prime}$ ). $\Delta k_{z}^{(0)}=k_{p}-2 k \cos \theta$ represents a constant term which must vanish to ensure phase matching. We ignore second order and higher terms in Eqs. (23) and (24) since the resulting overall frequency range is quite limited [see also Fig. [7. The central idea of this approach is to exploit the fact that, as expressed in Eqs. (23) and (24), whereas the longitudinal phase mismatch depends on the frequency sum $\nu_{s}+\nu_{i}$, the transverse phase mismatch depends on the frequency difference $\nu_{s}-\nu_{i}$. This means that while the contours of the longitudinal phase matching function $\phi_{z}\left(\Delta k_{z}\right)$ have negative unit slope those of the transverse phase matching function $\phi^{\perp}\left(\Delta k^{\perp}\right)$ have positive unit slope. Therefore, through an appropriate choice of the widths of the two functions (proportional to $L^{-1}$ and $w_{0}^{-1}$ respectively) the overall phase matching function can be made to be factorizable. The physical reason behind the positive slope of the transverse phase matching function is that transverse momentum conservation leads to the signal and idler photons propagating on opposite sides of the pump i.e. $\theta_{s}=-\theta_{i}$. Because this is a fundamental requirement of non-collinear degenerate PDC, this scheme (unlike that in ref. [4]) works at any central PDC wavelength where phase matching occurs.

When substituting Eq. (23) into Eq. (21) and Eq. (24) into Eq. (22), and multiplying the two resulting functions, there is a cross-term proportional to $\nu_{s} \nu_{i}$ in the exponential argument which is responsible for the non-factorizability of the phase matching function. Our approach is to let this term vanish, thus imposing a constraint on the crystal length $L$ and the beam diameter at the beam waist $w_{0}$. This constraint tells the experimenter, for a given crystal length, the required focusing strength needed in order to ensure a factorizable phase matching 
function:

$$
\frac{w_{0}}{L}=\frac{\sqrt{\gamma}\left(k_{p}^{\prime}-k^{\prime} \cos \theta\right)}{k^{\prime} \sin \theta}
$$

Note that there is a threshold bandwidth of the pump envelope which must be exceeded for the spectral uncorrelation of the state not to be destroyed by the strict spectral anti-corelation imposed by a narrow (or CW) pump:

$$
\sigma_{p}>\frac{\sqrt{2}}{\gamma L\left(k_{p}^{\prime}-k^{\prime} \cos \theta\right)}
$$

where $\sigma_{p}$ refers to the pump bandwidth defined in Sec.2. Let us look at a specific example involving a $1 \mathrm{~mm}$ long type-I BBO crystal with a cut angle of $\theta_{P M}=30.32^{\circ}$. When pumping such a crystal with an ultrafast pulsed pump centered at $\lambda_{p}=0.4 \mu \mathrm{m}$, degenerate noncollinear PDC is phase matched at propagation angles (within the crystal) of $\theta_{s}=3^{\circ}$ and $\theta_{i}=-3^{\circ}$. The condition in Eq. (25) gives a beam diameter of $w_{0}=287 \mu \mathrm{m}$. Note that with $w_{0} / L=0.287$ and $\theta=3^{\circ}$ we are in a regime where the approximation $w_{0} / L \gg \sqrt{\gamma} \sin ^{2} \theta$ is valid. Fig. [7]shows graphically the interplay of the longitudinal and transverse phase matching functions which combine to yield an uncorrelated state. Note that the downconverted photons are in this example emitted with a central wavelength of $\lambda=0.8 \mu \mathrm{m}$ so that they can be conveniently detected with silicon based detectors.
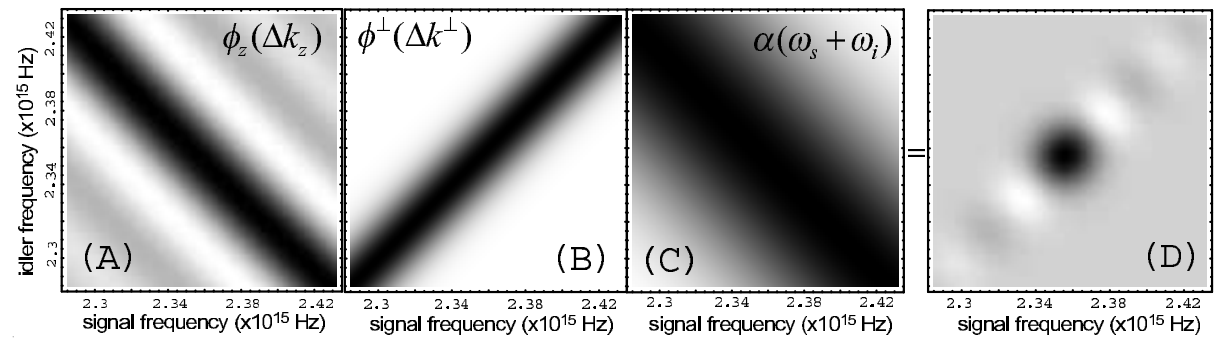

Fig. 7. An uncorrelated two-photon state can be synthesized by exploiting transverse momentum in the crystal. Shown here is the case of a type-I BBO crystal pumped at $400 \mathrm{~nm}$ with output modes at $\pm 3^{\circ}$. (A) shows the longitudinal phase matching function with negative unity contour slope, (B) shows the transverse phase matching function with unit contour slope, (C) shows the pump envelope function with a FWHM bandwidth of $10 \mathrm{~nm}$, (D) shows the product of the three previous functions, or the joint spectral amplitude, which is nearly spectrally uncorrelated. We can see that a narrow bandwidth for the pump envelope function would destroy the spectral uncorrelation.

\subsection{Engineering polarization entangled photon pairs without spectral correla- tions}

As discussed at the end of Sec. 2.3] a spectrally-uncorrelated polarization-entangled twophoton state has an appealing structure in that the spectral degree of freedom plays no role. Here we propose a straightforward adaptation of the technique described in the previous section for generating such a spectrally-uncorrelated polarization entangled state. This method is based on the ultrafast-pumped cascaded type-I source demonstrated by Nambu et al. 15. and on the technique designed to eliminate spectral correlations presented in Sec. 3.1 Two 
type-I crystals are placed sequentially, with a relative optic axis rotation of $90^{\circ}$ as shown in Fig. 8. A focused Gaussian pump beam is used such that the crystal length (assumed to be the same for both crystals) and beam waist diameter fulfil Eq. (25). The two blue pumps, polarized horizontally and vertically respectively, have a relative temporal delay in order to pre-compensate for group velocity mismatch as described by Nambu and co-workers [15. The photon-pairs generated can be written in terms of effective creation operators [see Sec. 2.3] as in Eq. (16). The state thus produced has the important property that spectral indistinguishability is guaranteed in any interference experiment.

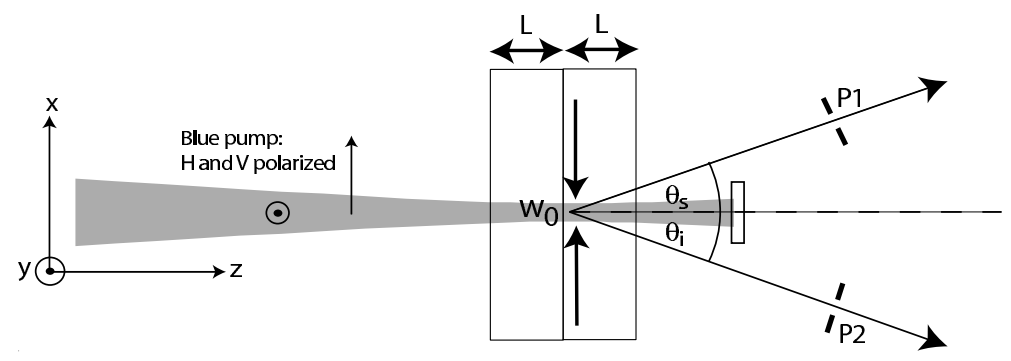

Fig. 8. Apparatus designed to generate a polarization-entangled frequency-uncorrelated twophoton pair.

\subsection{Engineering photon pairs with coincident frequencies}

There have been recent proposals for improved metrology by exploiting quantum entanglement. Giovannetti et al. [21] have proposed a quantum positioning scheme in which the temporal uncertainty in the time of arrival of $N$ photons, and therefore the uncertainty in the inferred position of the emitter with respect to the detectors, diminishes with the number of photons $N$ by a factor $\sqrt{N}$ faster than is possible with an analogous classical entanglementfree system. This positioning scheme necessitates an $N$-photon "frequency correlated" state where each of the emitted photons has an identical frequency. In the case of $N=2$ such a state can be written as:

$$
|\Psi\rangle=\int_{0}^{\infty} d \omega f(\omega)|\omega\rangle_{1}|\omega\rangle_{2}
$$

where $f(\omega)$ gives the relative frequency weighting. Such states, furthermore, exhibit an interesting dispersion cancellation effect to all orders as described by Erdmann and co-workers in Ref. 22] which opens up the exciting possibility of transmitting qubits over arbitrarily long dispersive fibers without decoherence. A technique has been proposed to generate such states as described by Giovanneti et al.223. This method relies on satisfying the group velocity matching condition 18 which unfortunately for most common $\chi^{(2)}$ materials occurs at longer $(>1 \mu m)$ wavelengths $\underline{4}$.

Here we propose a technique, based closely on the ideas described in the previous two sections, to generate frequency correlated states at wavelengths where Silicon-based single counting modules are well-developed. Consider the technique proposed in Sec. 3.1 and shown 


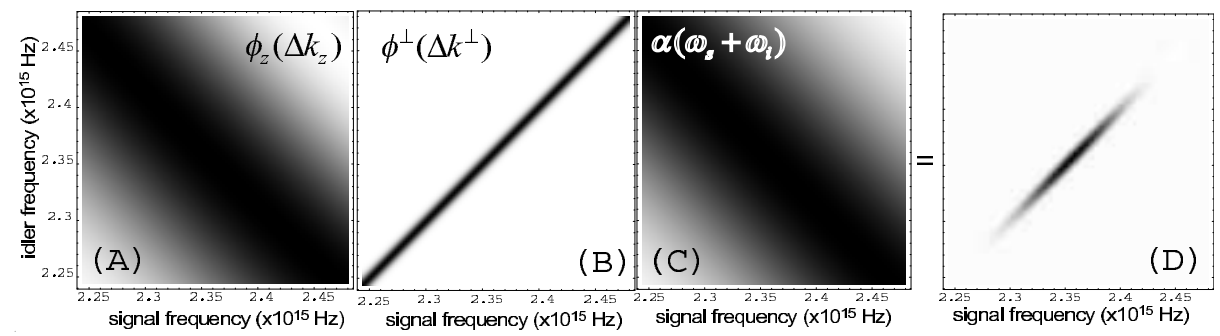

Fig. 9. A "frequency correlated" two-photon state can be synthesized by exploiting transverse momentum in the crystal. Shown here is the case of a $200 \mu \mathrm{m}$ long type-I BBO crystal pumped at $400 \mathrm{~nm}$ by a focused beam with spot size diameter $w_{0}=1 \mathrm{~mm}$ with output modes at $\pm 3^{\circ}$. (A) shows the longitudinal phase matching function with negative unity contour slope, (B) shows the transverse phase matching function with unit contour slope, $(\mathrm{C})$ shows the pump envelope function with a FWHM bandwidth of $15 \mathrm{~nm}$, (D) shows the product of the three previous functions, or the joint spectral amplitude, exhibiting frequency correlation. Note that a strictly frequency correlated state would have a vanishingly small width in the $\nu_{s}-\nu_{i}$ direction. The state shown in (D) represents a good approximation to such an ideal state.

schematically in Fig. 7 The basic idea behind this scheme is to let the longitudinal phasematching function [see Eq. (21)] have a relatively wide bandwidth while letting the transverse phasematching function have a relatively narrow bandwidth [see Eq. (22)]. A narrow-band longitudinal phasematching function can be obtained by using a short PDC crystal while a wide-band transverse phasematching function can be obtained by choosing a relatively large focusing spot. As was the case for the uncorrelated state, an ultrafast pump is required with sufficient bandwidth to encompass the spectral structure provided by the longitudinal and transverse phasematching functions. The condition thus imposed on the crystal length $L$ and focused beam spot size $w_{0}$ can thus be expressed as follows:

$$
\frac{w_{0}}{L} \gg \frac{\sqrt{\gamma}\left(k_{p}^{\prime}-k^{\prime} \cos \theta\right)}{k^{\prime} \sin \theta}
$$

which should be compared with the condition guaranteeing spectral uncorrelation [see Eq. (25)]. In addition Eq. (26) needs to be fulfilled to ensure that the pump has sufficient bandwidth. Note that a strict frequency correlated state would have zero width along the $\omega_{s}-\omega_{i}$ axis to ensure that the signal and idler photon frequencies are identical. We can get a good approximation to such a state by using a large focused beam spot size. Fig. 9 shows a specific example of the synthesis a frequency correlated state employing a $200 \mu \mathrm{m}$-long type-I BBO crystal with a cut angle of $\theta_{P M}=30.32^{\circ}$. When pumping such a crystal with an ultrafast pulsed pump centered at $\lambda_{p}=0.4 \mu \mathrm{m}$, degenerate non-collinear PDC is phase matched at propagation angles (within the crystal) of $\theta_{s}=3^{\circ}$ and $\theta_{i}=-3^{\circ}$. A focused beam spot size of $w_{0}=1 \mathrm{~mm}$ ensures that the condition expressed in Eq. 28$]$ is fulfilled yielding a frequency correlated two-photon source over the bandwidth imposed by the pump bandwidth and the crystal length as shown in Fig. 9(D). 


\section{Efficient generation of photon pairs}

In the preceding section, we simplified the analysis with the assumption that the downconversion output is collected from well defined directions, which corresponds to fixing the values of the transverse wave vectors in the joint two-photon probability amplitude. The task of engineering the complete modal characteristics of the down-converted photons has to include these spatial degrees of freedom as well. A promising route towards gaining control over the spatial structure of PDC is offered by non-linear waveguides, in which the downconversion process generates photon pairs in the modes supported by the waveguide structure. In this section, we review our recent experimental work on this subject.

Nearly all PDC experiments to date have used as source a nonlinear $\chi^{(2)}$ bulk crystal exploiting the principle of bi-refringent phase-matching. Observation of PDC in nonlinear waveguides incorporating the principle of quasi phase matching (QPM) has been the subject of several recent experiments [24, 25, 26, 27, 28]. Two of them [24, 25] consisted of detecting coincidences on the whole down-conversion signal divided by a 50:50 beam splitter. The third experiment [26] measured a Franson-type two-photon interference effect using an unbalanced Michelson interferometer. Recently, Gisin et al. have used PDC generated in a periodically poled lithium niobate (PPLN) waveguide to demonstrate time-bin entangled qubits [28. In an experiment by our own group [27] we aimed at separating all the photon pairs of interest into two different spatial paths with the help of a spectrographic setup. Using this technique, we were able to demonstrate the presence of strong correlations between photons of different frequencies. We treated one path as a trigger while collecting effectively all the conjugate photons in the second signal path. The quantity of primary interest in our experiment is thus the ratio of coincidences to single trigger counts, and the setup presented below can be viewed as a scheme for generating single "heralded" photons in the temporal slots defined by the firing of the trigger detector.

The experimental setup is depicted in Fig. 10. The output of a mode-locked Ti:Sapphire oscillator is first doubled in a type-I BBO crystal to generate blue pulses which are focused on the input face of a $1 \mathrm{~mm}$ long quasi-phase-matched $\mathrm{KTiOPO}_{4}$ (KTP) waveguide. The production and the characteristics of the sample used in our experiment have been described elsewhere [29]. The light power injected into the waveguide, measured before the objective, is about $22 \mu \mathrm{W}$. The bandwidth of the down-converted light for the parameters of our experiment far exceeds $100 \mathrm{~nm}$, and the down-converted photons are polarized parallel to the pump field. A spectrographic setup is used to separate the frequency anti-correlated signal and idler photons. The maximum ratio of coincidence counts to singles observed in our experiment is $18.5 \%$. This figure can be considered as the overall detection efficiency of the signal photons, including all losses from generation to detection. Further details of the experiment may be found in Ref. [27.

The highest ratio of coincidences to singles reported in a PDC experiment, exceeding $75 \%$ at $702 \mathrm{~nm}$, was observed by Kwiat et al. 31. It should be noted, however, that this figure provided by the authors entailed the subtraction of optical losses not due to the non-unit quantum efficiency of the detectors. The use of a long crystal $(10 \mathrm{~cm}$ long KDP crystal) introduced more stringent phase-matching conditions leading to strong spatial correlations between the down-converted photons.

The utilization of QPM means that because the pump, signal and idler photons can 


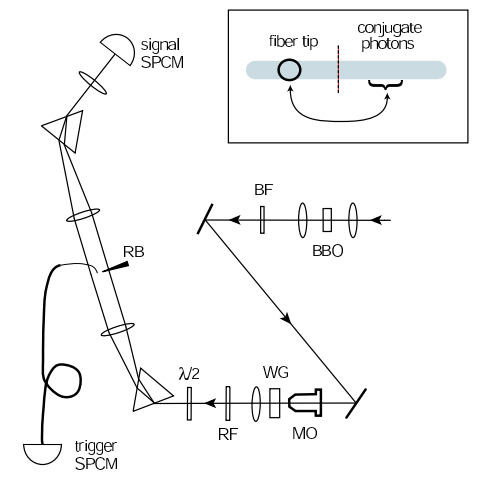

Fig. 10. Experimental setup for efficient generation of photon pairs using a KTP nonlinear waveguide.

have the same polarization, the diagonal elements of the $\chi^{(2)}$ tensor are accessible. Because diagonal elements are often higher in magnitude than non-diagonal elements, this leads to higher production rates. Furthermore, the fact that photon generation is constrained to the modes of the waveguide means that photons can be collected more efficiently as compared to the case of a bulk crystal, where photons are emitted in comparatively complicated spatial patterns. Our experiment shows that a nonlinear waveguide can be used as a single "heralded" photon source with high brightness while using a relatively short crystal and a relatively weak pump. Thus, nonlinear waveguides have the very important advantage that PDC can be generated economically as compared to bulk crystals. To illustrate this, Table 1 shows the economy figure of merit $R=R_{s} / L P$ for several recent PDC experiments where $R_{s}$ is the number of (single) photons detected per second, $L$ the length of the crystal and $P$ the pump power used. As can be seen, waveguide sources are associated with a value of $R$ several orders of magnitude higher that with bulk crystals.

Table 1. Generating correlated photon pairs efficiently.

\begin{tabular}{|r|r|r|r|r|r|}
\hline REF. & CRYSTAL & $P[\mathrm{~mW}]$ & $R_{s}[\mathrm{kHz}]$ & $\frac{R_{c}}{R_{s}}[\%]$ & $R\left[\frac{\mathrm{Hz}}{\mathrm{m} \cdot \mathrm{W}}\right]$ \\
\hline 31 & Type I 10cm KDP & .010 & 65 & 75 & $6.5 \times 10^{7}$ \\
30] & Type II 2mm BBO & 465 & 1250 & 26 & $2.7 \times 10^{6}$ \\
\hline 27 & 1mm KTP waveguide & .022 & 720 & 18.5 & $3.3 \times 10^{10}$ \\
\hline
\end{tabular}

\section{Application: Efficient linear optics quantum computation}

There have been recent proposals for building practical quantum computers using passive linear optical systems [1, 2]. Ralph et al. 2] developed a simplification of the original Knill, Laflamme and Milburn (KLM) [1] proposal which envisions the nonlinear sign-shift (NS) gate as a basic building block of quantum computers. They show that a C-NOT gate can be constructed from two such NS gates. Here we focus on the photonic engineering challenges that would be faced when implementing an NS gate using PDC sources.

The NS gate is shown in Fig. 111(A) and its operation is described in Ref. 2]. We assume the convention that a $\pi$ phase shift occurs only upon reflection from one side (indicated in 


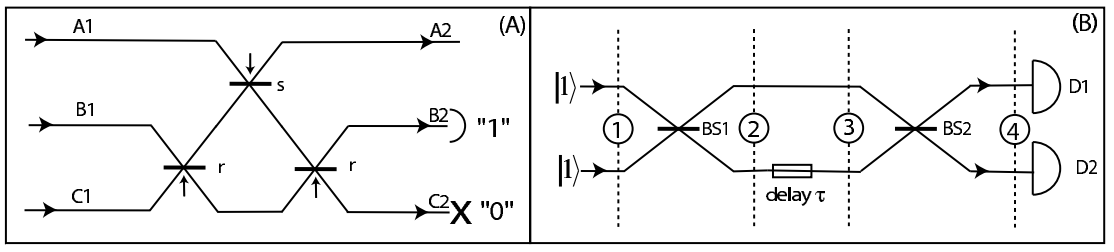

Fig. 11. (A) Non-linear sign shift (NS) gate. (B) HOMI Mach-Zendher interferometer.

Fig. 11(A) with a vertical arrow). Suppose the inputs in the ancillary channels $B 1$ and $C 1$ are $|1\rangle$ and $|0\rangle$ (where $|n\rangle$ is an $n$-occupancy Fock state) respectively and that the signal input in channel $A 1$ is occupied by the state $\left|\Psi_{\text {IN }}\right\rangle=\alpha|0\rangle+\beta|1\rangle+\gamma|2\rangle$. It is shown by Ralph et al. that the firing pattern: "1" photon in ancillary output $B 2$ and "0" photons in ancillary output $C 2$ implies that the $|2\rangle$ term has undergone a $\pi$ phase shift, so that the signal output at $A 2$ becomes: $\left|\Psi_{\text {OUT }}\right\rangle=\alpha|0\rangle+\beta|1\rangle-\gamma|2\rangle$. The gate is non-deterministic in the sense that this firing pattern occurs $25 \%$ of the time for an optimal choice of beamsplitters reflectivities $r$ and $s$ [see Fig. [11(A)]. Note that the NS gate necessitates a detector which can discriminate between 1 and 2 photons. The proposal in Ref. 32 may help in realizing such discrimination.

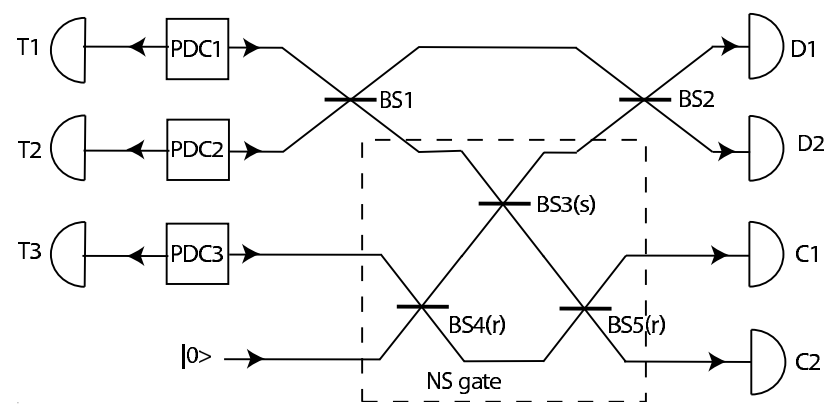

Fig. 12. An NS gate coupled to one arm of a HOMI Mach Zehnder interferometer in an implementation involving three PDC sources.

Let us turn our attention to the HOMI Mach-Zehnder interferometer (HOMI-MZ) depicted in Fig. 11(B). The two inputs are each occupied by a single photon coming for instance from a PDC crystal. The quantum interference at $B S 1$ turns the input state $\left|\Psi_{1}\right\rangle=|1\rangle|1\rangle$ into $\left|\Psi_{2}\right\rangle=(|0\rangle|2\rangle-|2\rangle|0\rangle) / \sqrt{2}$ [the subscripts label the interferometer stages as indicated in Fig. [11(B)]. If there is no phase difference between the two Mach Zehnder arms, $B S 2$ simply reverts the state back to the input i.e. $\left|\Psi_{4}\right\rangle=|1\rangle|1\rangle$, which leads to a coincidence event across the two outputs. However, if there is a phase difference of $\pi$ between the two arms, the state before $B S 2$ becomes $\left|\Psi_{3}\right\rangle=(|0\rangle|2\rangle+|2\rangle|0\rangle) / \sqrt{2}$, which remains unaltered by the second beam splitter. In this case, there is no coincidence event across the two outputs. Our approach is to employ this sensitivity to the phase difference between the two interferometer arms to test the action of the NS gate. 
We propose a practical implementation involving three PDC sources, so that six photons are emitted simultaneously. The proposed apparatus is shown in Fig.12 an NS gate is coupled to one arm of a HOMI-MZ with three stacked PDC crystals acting as a source of three photons conditioned on the firing of the triggers $T 1, T 2$ and $T 3$. When the NS gate triggers (i.e. "1" photon is recorded at detector $C 1$ and none at detector $C 2$ ) the lower interferometer arm undergoes a $\pi$ phase shift, which means that the photons emerging at $D 1$ and $D 2$ are both in the same (but undetermined) channel so that no $D 1, D 2$ coincidence event occurs. Therefore, we may be convinced that the simultaneous firing of detectors $T 1, T 2, T 3, D 1, D 2, C 1$ is an impossibility. A different way of saying this is that a $T 1, T 2, T 3, D 1, D 2, C 1$ coincidence event implies that the NS gate did not fire. We use this fact to test the performance of the NS gate. If the probability of obtaining such a six-fold coincidence event is non-zero, the correlation between the $C 1, C 2$ and $D 1, D 2$ firing patterns is imperfect.

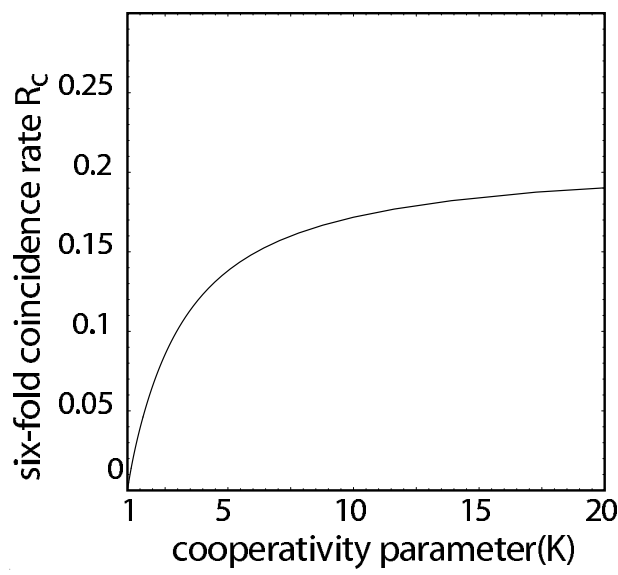

Fig. 13. Relationship between the six fold $T 1 T 2 T 3 D 1 D 2 C 1$ coincidence rate and the cooperativity parameter. In the ideal case where the firing patterns at $\mathrm{C} 1$ and $\mathrm{C} 2$ are perfectly correlated to those at D1 and D2, the coincidence rate must vanish. Note that for $K>1$, i.e. for a source which exhibits spectral correlations the NS gate does not operate ideally.

The system described here involves multiple-source quantum interferometry of the kind described in Sec. 3. We would therefore expect the arguments about indistinguishability in the form of spectral correlation to apply so that the system should work perfectly only in the case where a single Schmidt mode is active (i.e. $K=1$ ) in each of the three PDC sources. In order to model this apparatus, we assume that the three PDC sources are described by a simplified type-I joint spectral amplitude as given by Eq. (6). The six-fold $T 1, T 2, T 3, D 1, D 2, C 1$ coincidence rate is given by $\left.R_{c}=\int \ldots \int d t_{1} \ldots d t_{6}\left|\hat{E}_{1}^{(-)}\left(t_{1}\right) \ldots \hat{E}_{6}^{(-)}\left(t_{6}\right)\right| \Psi\right\rangle\left.\right|^{2}$ where $\hat{E}_{j}^{(-)}(t)$ with $j=1-6$ are the electric field operators at each of the six output channels and take into account the various beam-splitter transformations. The quantum state is assumed to be given by: $|\Psi\rangle=\int \ldots \int d \omega_{1} \ldots d \omega_{6} f\left(\omega_{1}, \omega_{2}\right) f\left(\omega_{3}, \omega_{4}\right) f\left(\omega_{5}, \omega_{6}\right) \hat{a}_{1}^{\dagger} \ldots \hat{a}_{6}^{\dagger} \mid$ vac $\rangle$ where each function $f\left(\omega_{i}, \omega_{j}\right)$ is given according to Eq. (6). We further assume that the beamsplitter reflectivities are given by [2]: $r=(4-2 \sqrt{2})^{-1}$ and $s=(\sqrt{2}-1)^{2}$. Such a calculation yields the result expressed in the plot of coincidence rate vs. cooperativity parameter in Fig. 13. Note that 
a zero coincidence rate is obtained, as expected, only in the case where there is no spectral correlation.

\section{Conclusions}

Designing PDC sources with characteristics optimized for quantum information processing applications presents a highly nontrivial task. We have shown in this paper that a careful analysis of the spatio-temporal characteristics of the down-conversion process yields possible routes towards practical solutions to this problem. These results, combined with the possibility of engineering non-linear two-photon states by using waveguide structures, open the prospects for the next generation of down-conversion sources with the characteristics tailored specifically for quantum information experiments. We have illustrated the importance of source engineering with a discussion of optical implementations of simple quantum logic circuits such as the nonlinear phase shift gate.

\section{Acknowledgements}

We have become aware since preparing this manuscript that W.P.Grice and Y. Kim have arrived at similar conclusions to the ones presented in Sec. 2.3]33. The authors acknowledge insightful discussions with W.P. Grice, M. Teich, A. Sergienko and M. Raymer. This work was partially supported by ARO-administered MURI grant number DAAG-19-99-1-0125 and by the National Science Foundation (NSF).

\section{References}

1. E. Knill, R. LaFlamme and G.J Milburn (2001), A scheme for efficient quantum computation with linear optics Nature, 409, pp. 46-52

2. T.C. Ralph, A.G. White, W.J. Munro and G.J Milburn(2001), Simple scheme for efficient linear optics quantum gates Phys. Rev. A, 65, pp. 012314

3. C.K. Law, I.A. Walmsley and J. H. Eberly (2000), Frequency Entanglement: Effective Finite Hilbert Space and Entropy Control Phys. Rev. Lett., 84, pp. 5304-5307

4. W.P. Grice, A.B. U'Ren and I.A. Walmsley (2001), Eliminating frequency and space-time correlations in multiphoton states Phys. Rev. A, 64, pp. 063815

5. H. Huang and J.H. Eberly (1993), One-Quantum Pulse Shapes In Photon Pair Generation J. Mod. Opt., 40, pp. 915-930

6. C. Santori, D. Fattal. J. Vuckovic and Y. Yamamoto (2002), Indistinguishable photons from a single-photon device Nature, 419, pp. 594-597

7. I. Lvovsky, H. Hansen, T. Aichele, O. Benson, J. Mlynek and S. Schiller (2001), Quantum State Reconstruction of the Single-Photon Fock State Phys. Rev. Lett., 87,pp. 050402

8. W.P. Grice and I.A. Walmsley (1997), Spectral information and distinguishability in type-II downconversion with a broadband pump Phys. Rev. A, 56, pp. 1627

9. W.P. Grice. (1997) Interference and Indistinguishability in Ultrafast Spontaneous Parametric Downconversion Ph.D. thesis, University of Rochester

10. C.K. Hong, Z.Y. Ou and L. Mandel (1987), Measurement of Subpicosecond Time Intervals Between 2 Photons by Interference Phys. Rev. Lett., 59, pp. 2044-2046

11. A.B. U'Ren, E. Mukamel, K. Banaszek and I. Walmsley (2003) Photon managment for quantum information processing To appear, Royal Society proceedings

12. P.G. Kwiat, K. Mattle,H . Weinfurter H. and A. Zeilinger (1995) New High-Intensity Source of Polarization-Entangled Photon Pairs Phys. Rev. Lett. 75, pp. 4337-4340 
13. P.G. Kwiat, E. Waks, A.G. White, I. Appelbaum and P.H. Eberhard (1999) Ultrabright source of polarization-entangled photons Phys. Rev. A 60 pp. 773

14. S.L. Braunstein and A. Mann (1995) Measurement of the Bell operator and quantum teleportation Phys. Rev. A 51 pp. R1727R1730

15. Y. Nambu, K. Usami, Y. Tsuda, K. Matsumoto and K. Nakamura(2002) Generation of polarization-entangled photon pairs in a cascade of two type-I crystals pumped by femtosecond pulses Phys. Rev. A 66 pp. 033816

16. Y.H. Kim, and W.P. Grice (2002) Generation of pulsed polarization-entangled two-photon state via temporal and spectral engineering J. Mod Opt., 49 pp. 2309-2323

17. Y.H. Kim, S.P. Kulik, M.V. Chekhova, W.P. Grice, and Y. Shih(2003) Experimental entanglement concentration and universal Bell-state synthesizer Phys. Rev. A, 67, pp. 010301

18. T.E. Keller and M.H. Rubin (1997) Theory of two-photon entanglement for spontaneous parametric down-conversion driven by a narrow pump pulse Phys. Rev. A, 56, pp. 15341541

19. F. De Martini, G. di Giuseppe, S. Padua (2001) Multiparticle quantum superposition and stimulated entanglement by paritive selective amplification of entangled states Phys. Rev. Lett., 87, pp. 150401

20. A. Lamas-Linares, J.C. Howell JC and D. Bouwmeester (2001) Stimulated emission of polarizationentangled photons Nature, 412 pp. 6850

21. V. Giovannetti, S. Lloyd and L. Maccone (2001) Quantum-enhanced positioning and clock synchronization Nature, 412 pp. 417-419

22. R. Erdmann, D. Branning, W. Grice and I. Walsmley (2000) Restoring dispersion cancellation for entangled photons produced by ultrashort pulses Phys. Rev. A, 62 pp. 53810

23. V. Giovannetti, L Maccone, J.H. Shapiro and F.N.C. Wong (2002) Generating entangled twophoton states with coincident frequencies Phys. Rev. Lett., 88 pp. 183602

24. V. Pruneri, G. Bonfrate, P.G. Kazansky, H. Takebe, K. Morinaga, M. Kohno, K. Kuwasaki and T. (1999), High second-order optical nonlinearities in thermally poled sol-gel silica Appl. Phys. Lett., 74 , pp. $2578-2580$

25. Tanzilli, H. De Riedmatten, W. Tittel, H. Zbinden, P. Baldi, M. De Micheli , D.B. Ostrowsky and N. Gisin (2001), Highly efficient photon-pair source using periodically poled lithium niobate waveguide Electron. Lett., 37, pp. 26-28

26. K. Sanaka, K. Kawahara and T. Kuga (2001), New high-efficiency source of photon pairs for engineering quantum entanglement Phys. Rev Lett., 86, pp. 5620-5623

27. K. Banaszek, A. U'Ren and I. Walmsley (2001), Generation of correlated photons in controlled spatial modes by downconversion in nonlinear waveguides Opt. Lett., 26, pp. 1367-1369

28. R.T. Thew, S. Tanzilli, W. Tittel, H. Zbinden and N. Gisin (2002), Experimental Investigation of the robustness of partially entangled qubits over $11 \mathrm{~km}$ Phys. Rev. A, 66, pp.062304

29. M. G. Roelofs, A. Suna, W. Bindloss, and J. D. Bierlein (1994), Characterization of Optical WaveGuides in Ktiopo4 By 2nd-Harmonic Spectroscopy J. Appl. Phys., 76, pp. 4999-5006

30. Kurtsiefer, M. Oberparleiter and H. Weinfurter (2001), High-efficiency entangled photon pair collection in type-II parametric fluorescence Phys. Rev. A, 64, pp. 023802

31. P.G. Kwiat, A.M. Steinberg, R.Y. Chiao (1993), High-Efficiency Single-Photon Detectors Phys. Rev. A, 48, pp. R867

32. K. Banaszek and I. Walmsley (2003)Photon counting with a loop detector Opt. Lett., 28, pp. 52

33. Y.H.Kim and W.P. Grice (2003) Reliability of the beamsplitter based Bell-state measurement quant-ph/0304066

\section{Appendix A}

While carrying out a Schmidt decomposition analytically for an arbitrary two-photon state is non-trivial, for certain classes of states it may be achieved. We will find the following identity 
useful:

$$
\begin{array}{r}
\exp \left[-\frac{1+\mu^{2}}{2\left(1-\mu^{2}\right)}\left(\alpha_{1}^{2} x_{1}^{2}+\alpha_{2}^{2} x_{2}^{2}\right)+\frac{2 \alpha_{1} \alpha_{2} \mu x_{1} x_{2}}{1-\mu^{2}}\right]= \\
\sqrt{1-\mu^{2}} \sum_{n=0}^{\infty} \mu^{n} u_{n}\left(\alpha_{1} x_{1}\right) u_{n}\left(\alpha_{2} x_{2}\right)
\end{array}
$$

valid for $\mu \neq 0$ and where $u_{n}(x)=\left(2^{n} n !\right)^{-\frac{1}{2}} H_{n}(x) \exp \left(-x^{2} / 2\right), H_{n}(x)$ being the $n^{\text {th }}$ order Hermite polynomial. Any two photon state characterized by a spectral amplitude $f\left(\omega_{s}, \omega_{i}\right)$ which can be written in the form of the left-hand-side of Eq. (A.1) can be decomposed into its Schmidt functions with the help of this identity. Comparing Eq. (A.1) with Eq. (2), we can write down an expression for the eigenstates $\lambda_{n}$ :

$$
\lambda_{n}=\left(1-\mu^{2}\right) \mu^{2 n}
$$

By substituting Eq. (A.2) into Eq. (3), we obtain an expression for the cooperativity parameter:

$$
K=\frac{1+\mu^{2}}{1-\mu^{2}}
$$

The two-photon state characterized by the idealized type-I two-photon state introduced in Sec. 2 [see Eq. [6] ] belongs to the class of sates decomposable through Eq. (A.1). Carrying out the decomposition we find that:

$$
\mu=1+\left(\frac{\sigma}{\sigma_{F}}\right)^{2}-\sqrt{2\left(\frac{\sigma}{\sigma_{F}}\right)^{2}+\left(\frac{\sigma}{\sigma_{F}}\right)^{4}}
$$

We can now obtain an expression for the cooperativity parameter for the type-I idealized source in terms of the source parameters $\sigma$ and $\sigma_{F}$ by substituting Eq. (A.4) into Eq. (A.3). Such an expression was used to generate the plots in Fig. 3 . 